Proc. Indian Acad. Sci. (Chem. Sci.), Vol. 96, No. 6, April 1986, pp. 391-409.

(C) Printed in India.

\title{
The synthesis, crystal growth, and characterization of nonstoichiometric magnetite
}

\author{
J M HONIG \\ Department of Chemistry, Purdue University, West Lafayette, Indiana 47907, USA
}

\begin{abstract}
A review is provided concerning the thermodynamic and electrical characteristics of nonstoichiometric magnetite, $\mathrm{Fe}_{3(1-\delta)} \mathrm{O}_{4}$. Great stress is placed on the need for careful preparation and appropriate annealing of single crystal specimens. Information needed to prepare material of a given $\delta$ value is provided. For $\delta \leqslant 0.0039$ magnetite undergoes a firstorder phase change at the Verwey transition; for $\delta>0-0039$ the change is of second or higher order. Corresponding types of changes are encountered in resistivity and Seebeck coefficient measurements. Some aspects of the Verwey transition are discussed.
\end{abstract}

Keywords. Magnetite; metal-insulator transitions; heat capacity measurements; electrical properties.

\section{Historical commentary}

Magnetite has been recognized as an important substance since antiquity; Plinius and Dioskorides were among the early natural philosophers interested in the properties of this material which is referred to under the generic name of lodestone. It was the magnetic properties of this class of minerals that held the interest of many natural philosophers for centuries. More systematic investigations were initiated by GayLussac (1811, 1812), Dalton (1816), and Berzelius (1826). The fundamental physical properties of this material were more firmly established by numerous investigators in the period 1850-1925. In particular, the scientific analysis of the ferrimagnetic properties of magnetite was placed on a sound footing only after the fundamental analysis of this phenomenon by Néel (1948).

The first report of an anomaly in $\mathrm{Fe}_{3} \mathrm{O}_{4}$ occurring in the range $113-117 \mathrm{~K}$ seems to have been published in 1926 by Parks and Kelley (1926), on the basis of specific heat measurements. This was followed by further exploratory investigations by Okamura (1932) of several other properties, all of which exhibited anomalies in the 100-125 K range. Verwey (1939) published a seminal article in which he directed attention to a discontinuity in electrical resistivity near $120 \mathrm{~K}$ as a manifestation of a transition that now bears his name. Already in this first paper the nature and temperature of the transformation was shown to be sensitive to small changes in the oxygen/iron ratio in $\mathrm{Fe}_{3} \mathrm{O}_{4}$, but his recognition of the need to control sample compositions was not properly followed up in most of the work published on the subject since that time. As a consequence, there is considerable diversity in the reported experimental results; this, in turn, has produced many different theories designed to elucidate the nature of the Verwey transition. It has only recently been properly appreciated that the phenomenon is intimately linked to the mixed valence properties of the cation, i.e., to the ratio of iron present in the two distinct oxidation states +2 and +3 . Thus, if one wishes to understand the fundamentals of the Verwey transition it is important to avoid any conditions under which the $\mathrm{Fe}^{2+} / \mathrm{Fe}^{3+}$ ratio is inadvertently altered from the desired 
value, by changes in the $\mathrm{O} / \mathrm{Fe}$ ratio or by the presence of impurities. This, in turn, calls for precise control over sample preparation techniques. Since sufficient care with respect to these matters has not always been exercised in the past, a renewed study of even the most fundamental properties with adequately prepared specimens has become extremely important. One such data have been put on a firm footing the number of viable theoretical models describing the transition will also be greatly reduced.

In light of the above, we first discuss methods of sample preparation and characterization that lead to specimens of adequate perfection. We then describe a series of recent studies which shed new light on the phenomenology of the Verwey transition. Lastly, we comment briefly on some implications of these findings. For earlier reviews of the status of the field the reader is referred to articles by Mott (1979, 1980 ) and by Honig (1982).

\section{Preparation of specimens}

$\mathrm{Fe}_{3} \mathrm{O}_{4}$ or $\mathrm{Fe}_{2} \mathrm{O}_{3}$ is available commercially in powdered form with low levels of impurities. The first task facing the investigator is the growth of single crystals by a technique which avoids sample contamination. A very effective method by which sizeable crystals may be grown is the skull-melting operation shown schematically in figure 1a (Harrison and Aragón 1978; Harrison et al 1983). The output of a $50 \mathrm{kw} \mathrm{rf}$ generator operating at $3 \mathrm{MHz}$ is routed to a coil wound around a copper crucible, depicted in figure $1 \mathrm{~b}$. The vertical fingers and sections of the split base are individually water-cooled and spaced sufficiently far apart to permit penetration of the electromagnetic field into the sample space; eddy currents through the metal container are also minimized by this arrangement.

As for any metal oxide, it is essential to control the oxygen fugacity above the specimens to ensure that the correct phase is obtained. One method for achieving this objective, both during crystal growth and in subsequent annealing processes, is to expose the sample to a $\mathrm{CO} / \mathrm{CO}_{2}$ gas mixture which is circulated above the specimen. At any particular temperature the equilibrium between these components establishes a definite oxygen fugacity through the reaction

$$
\mathrm{CO}_{2}=\mathrm{CO}+(1 / 2) \mathrm{O}_{2} \text {. }
$$

The oxygen fugacity, $f_{\mathrm{O}_{2}}$, is given by

$$
K=\left(f_{\mathrm{Co} /} / f_{\mathrm{CO}_{2}}\right) f_{\mathrm{O}_{2}}^{1 / 2},
$$

where the equilibrium constant $K$ is fixed at any specified temperature; the ratio $f_{\mathrm{CO}} / f_{\mathrm{CO}_{2}}$ is adjusted experimentally through control of the relative rates of flow of these two gases into the chamber. At a given temperature the oxygen fugacity is then uniquely specified. One should note that according to figure 2 , at volume flows for which the percentage of $\mathrm{O}_{2}$ in the gas mixture ranges from 100 to $94.3 \%$, one remains inside the stability limits of the magnetite phase; the various oxygen fugacity curves change with temperature in a manner approximately parallelling the change of the $f_{\mathrm{O}_{2}}$ curves corresponding to the phase boundaries.

During crystal growth one encounters the problem that $\mathrm{Fe}_{3} \mathrm{O}_{4}$ melts incongruently; because of their different $\mathrm{Fe}^{2+} / \mathrm{Fe}^{3+}$ ratios, liquid and solid magnetite at the melting point require different oxygen partial pressures for equilibration. It was found 

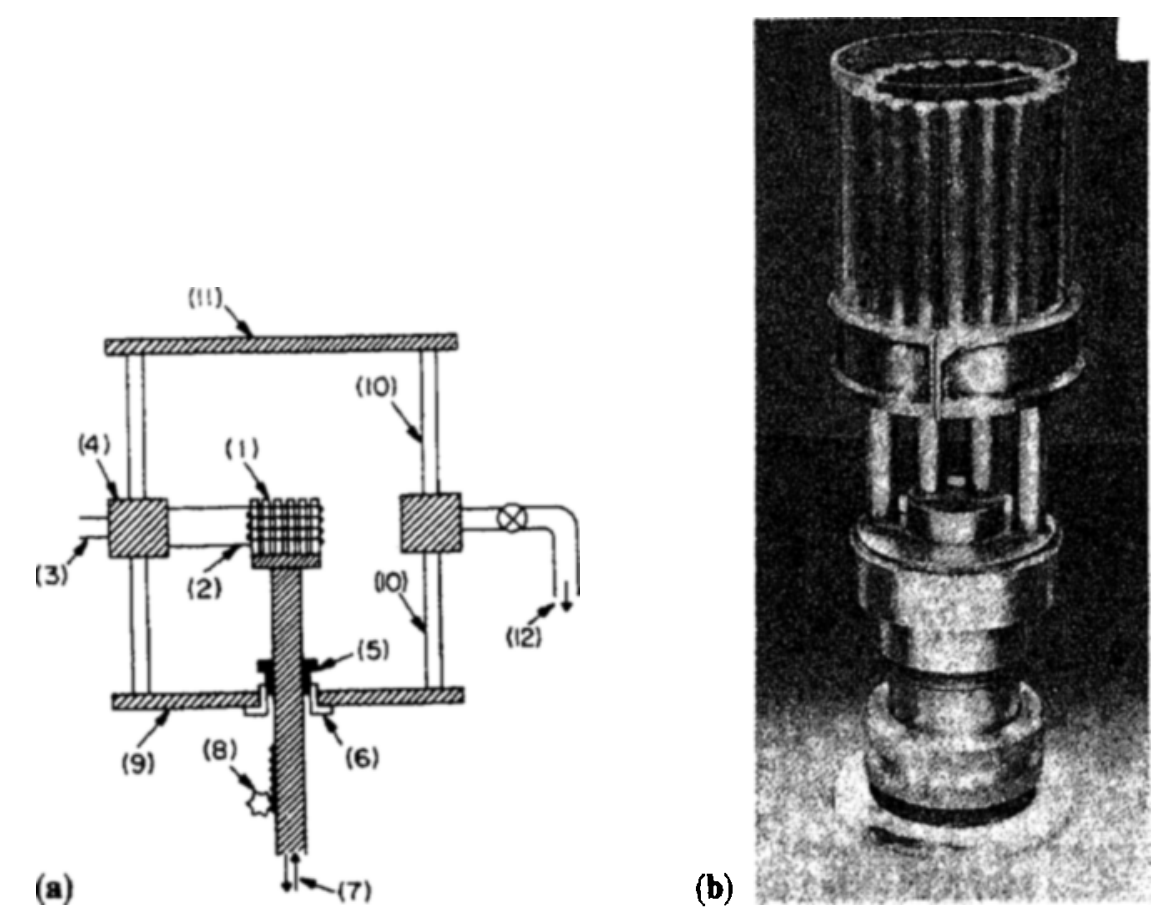

Figure 1(a). Schematic diagram of skull melter. (b). Photograph of a water-cooled copper container for use with skull melter.

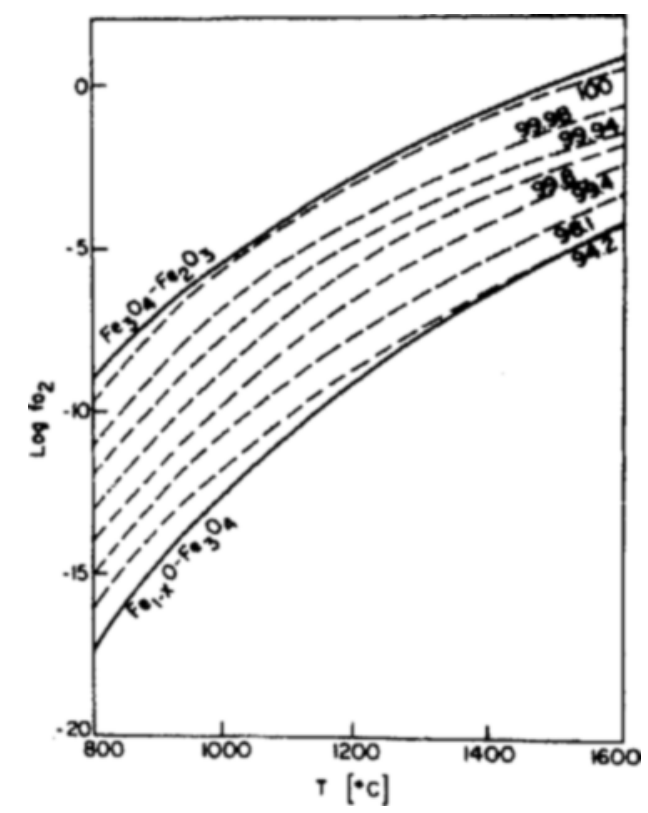

Figure 2. Phase stability diagram for magnetite. The solid boundaries depict the oxygen fugacities required at the magnetite-wüstite and magnetite-hematite phase boundaries. The dashed lines show oxygen fugacities achieved with several $\mathrm{CO}_{2} / \mathrm{CO}$ mixtures; figures quoted pertain to the $\mathrm{CO}_{2} /\left[\mathrm{CO}+\mathrm{CO}_{2}\right]$ content of the circulating gases. 
empirically that boules containing single crystals could be grown by use of $100 \% \mathrm{CO}_{2}$ as the circulating gas; however, the materials so obtained tend to be cation deficient and nonuniform in composition. This difficulty can be remedied by subsequent subsolidus annealing as described later; in the course of this process the proportions of $\mathrm{CO}$ and $\mathrm{CO}_{2}$ have to be fixed at prescribed values discussed below.

The melting process itself is initiated by placing a graphite ring on top of the $\mathrm{Fe}_{3} \mathrm{O}_{4}$ charge; the ring readily couples to the rf power supplied through the coils and thus heats contiguous regions of the powder sufficiently so that these, in turn, couple to the electric power. An avalanching process ensues until all the material becomes molten except for a layer of sintered material adjacent to all water-cooled surfaces of the copper container. This thin skull forms the 'crucible' for the molten $\mathrm{Fe}_{3} \mathrm{O}_{4}$ mass; the graphite susceptor burns off in the mildly oxidizing atmosphere above the sample. There is provision to add more powder by use of a hopper on top of the unit shown in figure 1a. A rack and pinion device lowers the molten mass through the coil to a cooler zone, thus allowing all the material to freeze slowly, and to form a solid boule. The latter then is opened up; single crystal magnetite ranging from less than $0.5 \mathrm{~g}$ to more than $20 \mathrm{~g}$ (and to $70 \mathrm{~g}$ on one occasion) may then be removed from the centre.

\section{Annealing of specimens}

While crystals prepared as described above appear to be of high quality to the unaided eye they are generally quite nonuniform in their $\mathrm{O} / \mathrm{Fe}$ ratio even within the same single crystal because of the incongruent melting problem described earlier and because of the severe thermal gradients that exist in the cooling process.

To circumvent the difficulty the single crystal nuggets are oriented and sliced to obtain samples of desired dimensions. Specimens so produced are then annealed at temperatures below their melting point. The annealing requirements involve two determinations: the appropriate oxygen fugacity to which the sample is to be subjected during the reheating process, and the length of time required to achieve uniformity of composition during that particular subsolidus anneal.

The oxygen fugacity is set by the desired degree of deviation $\delta$ from ideal magnetite stoichiometry to achieve the specified composition $\mathrm{Fe}_{3(1-\delta)} \mathrm{O}_{4}$. The appropriate thermodynamic analysis (Aragón et al 1982) is based on the following two equations (Flood and Hill 1957) that govern the equilibration processes of magnetite with oxygen gas:

$$
\mathrm{Fe}^{2+}+\frac{1}{4} \mathrm{O}_{2}=\mathrm{Fe}^{3+}+\frac{1}{2} \mathrm{O}^{2-}+\frac{3}{8} \mathrm{~V},
$$

and

$$
\frac{3}{\mathrm{I}} \mathrm{I}+\mathrm{Fe}^{2+}+\frac{1}{4} \mathrm{O}_{2}=\mathrm{Fe}^{3+}+\frac{1}{2} \mathrm{O}^{2-}
$$

Here $V$ is a vacancy in the cation sublattice and $I$ is an interstitialcy arising from the formation of Frenkel defect pairs. These two entities can recombine to yield a normal cation

$$
V+I=0
$$

An appropriate thermodynamic analysis (Aragon and Mcallister 1982) yields the 
following relation between the oxygen fugacity $f_{\mathrm{O}_{2}}$ and $\delta$ :

$$
f_{O_{2}}^{1 / 4}=\frac{1}{K_{2}}\left\{2+\frac{24 \delta}{1-8 \delta-\frac{\delta}{2}-\frac{1}{2}\left[\delta^{2}+4 K_{1}\right]^{1 / 2}}\right\}\left\{\frac{\delta}{2}+\frac{1}{2}\left(\delta^{2}+4 K_{1}\right)^{1 / 2}\right\}^{3 / 8}
$$

wherein

$$
K_{1}=n_{v} n_{d} / n_{\Sigma}^{2},
$$

is the equilibrium constant for the formation of Frenkel defect pairs, and

$$
K_{2}=\frac{n_{\mathrm{Fe}^{3+}}}{n_{\mathrm{Fe}^{2+}}} f_{\mathrm{O}_{2}}^{-1 / 4}\left(\frac{n_{\mathrm{v}}}{n_{\mathrm{E}}}\right)^{3 / 8} .
$$

In the above $n_{v}, n_{l}, n_{\Sigma}$ are the mole numbers of cation vacancies, of cations located interstitially, and of total cations, respectively. The equilibrium constants $K_{1}$ and $K_{2}$ can be evaluated from the carefully executed set of thermogravimetric experiments (Dieckmann 1982) by the determination of $\delta$ as a function of $f_{\mathrm{O}_{2}}$ at several temperatures while the sample remained equilibrated with the ambient. It was found that

$$
\begin{aligned}
& \log K_{2}=\frac{4456}{T}-2.64, \\
& \log K_{1}=\frac{-10740}{T}-1.23 .
\end{aligned}
$$

The dependence of $\log f_{\mathrm{O}_{2}}$ on $10^{3} / T$ is shown in figure 3 for several selected $\delta$ values within the magnetite-hematite and magnetite-wüstite phase boundaries shown on the diagram. One should note in particular that the stability curves corresponding to $\delta>0$ and $\delta<0$ are concave and convex respectively; thus, they ultimately cross the boundary lines delineating the phase stability limits for magnetite. Therefore, if one gradually cools a specimen enclosed in a container, so as to maintain the system isostoichiometric (i.e. at a fixed composition), one runs the risk of ending up with a two-phase mixture

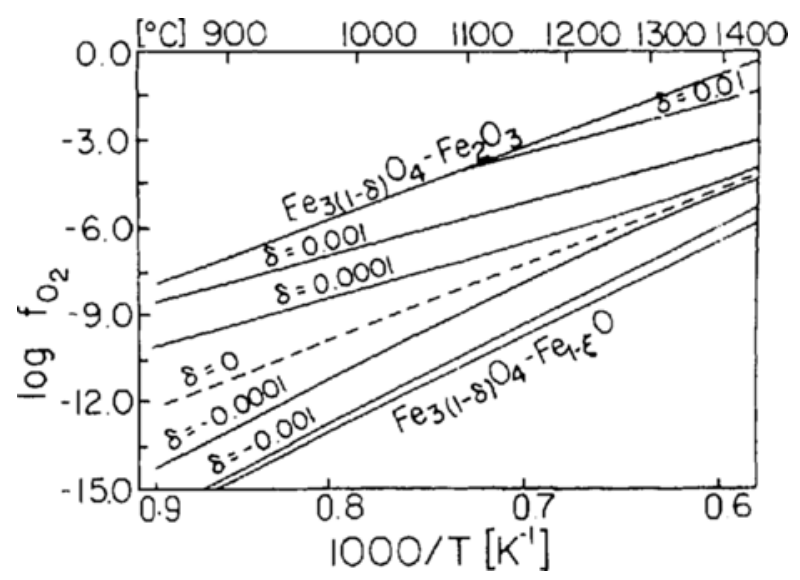

Figure 3. Intrinsic oxygen fugacity for single crystalline $\mathrm{Fe}_{3\{1-8\}} \mathrm{O}_{4}$ and stability fieid boundaries as a function of inverse temperature. The dashed line represents the oxygen fugacity in equilibrium with stoichiometric magnetite. 
unless the process is kinetically hindered; this problem may usually be avoided by a rapid quench. It is, however, necessary to check samples for the absence of extraneous phases (see below) after any cooling process even when the latter is carried out with a totally encapsulated magnetite sample.

In figure 4 is shown the variation of $\delta$ versus $\log f_{\mathrm{O}_{2}}$ at $1400^{\circ} \mathrm{C}$ as calculated from (6)-(10); the curves for the range $1300^{\circ} \mathrm{C}$ to $900^{\circ} \mathrm{C}$ are similar in appearance but the maximum excursions from $\delta=0$ become considerably smaller, as is also evident from inspection of figure 3 . One thus has available all the information required for the preparation of magnetite samples of a specific stoichiometry within the limitations imposed by the requirements of maintaining single-phase samples.

There then remains the question of the length of the anneal time needed to ensure a uniformity of composition within the bulk of the sample. This matter is addressed by examination of the diffusion rates of the cations in response to the addition or subtraction of oxygen in the lattice during equilibration. The requisite information is contained in figure 5 which represents a concentration profile for the motion of vacancies through a semiinfinite slab. The parameter associated with each curve is specified by $D t / l^{2}$, where $l$ is the half-thickness of the slab, $t$ is the time, and $D$ $\simeq D_{\mathrm{Fe}}^{*} /[V]$ is the vacancy diffusion constant, related as shown to the $\mathrm{Fe}$ tracer diffusion constant $D_{\mathrm{Fe}}^{*}$ and to the cation vacancy concentration $[V]$. The requisite $D_{\mathrm{Fe}}^{*}$ values are available from a study of Dieckmann and Schmalzried (1977); based on these results, typical times required for sample homogenization are shown as part in the figure caption. At first glance it appears highly desirable to operate at $1400^{\circ} \mathrm{C}$ so as to minimize the time for achieving a uniform sample composition. However, this then also requires an extremely fast quench so as not to disturb the uniformity of composition during the cooling process. Thus, it is easier to operate at $1000^{\circ} \mathrm{C}$ where the quenching process does not present a problem. On the other hand, as already mentioned, the maximum value of $\delta$ increases with rising temperatures; thus, to reach the larger values of $\delta$ it is necessary to carry out anneals at $1400^{\circ} \mathrm{C}$. As is seen from the figure, homogenization of a slab is complete in a time scale of minutes for samples whose thickness is in the mm range. This means that, even for very fast quenches, the surface layers change in composition as the sample cools. An appropriate operating procedure

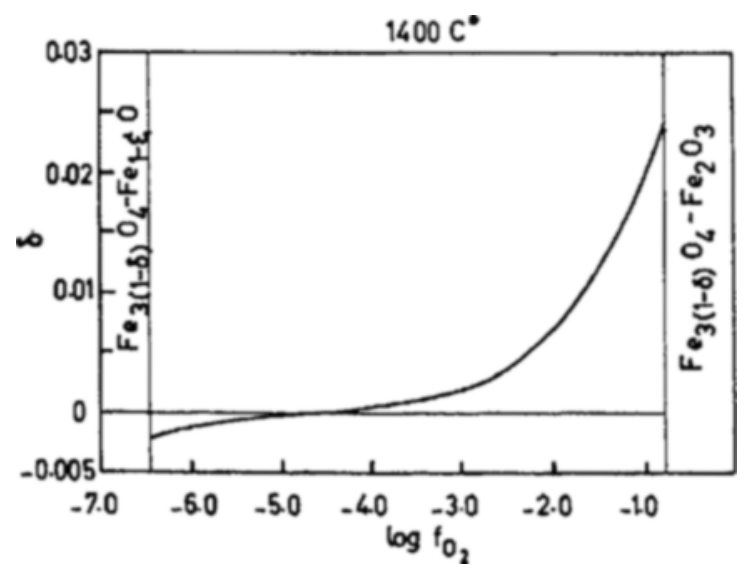

Figure 4. Variation of parameter $\delta$ with $\log f_{\mathrm{O}_{2}}$ at $1400^{\circ} \mathrm{C}$. 


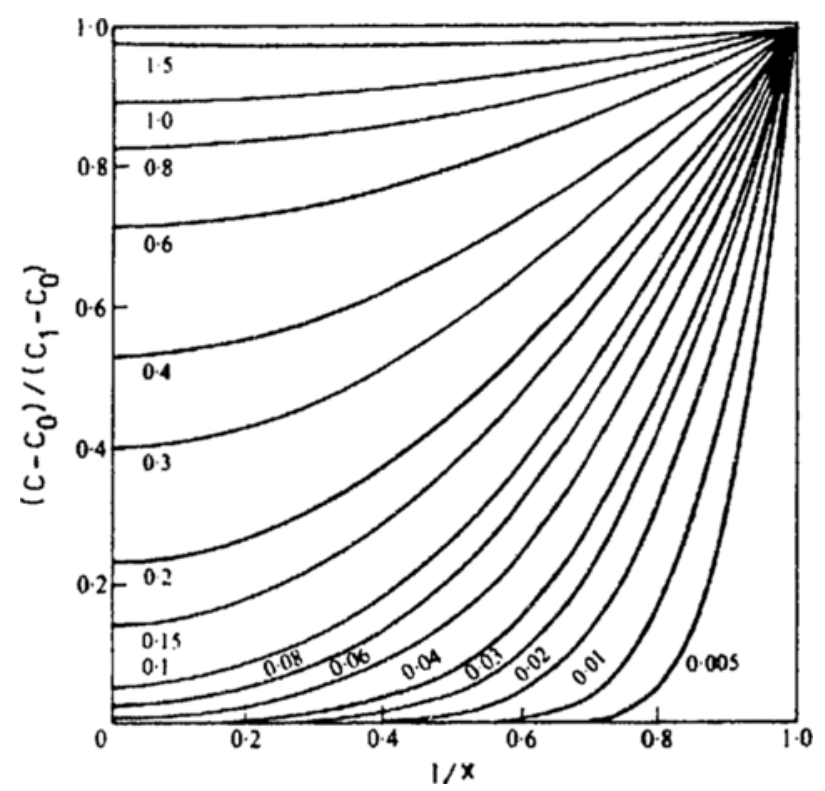

Figure 5. Concentration profiles for bulk diffusion. $C$ represents the concentration of the diffusing species; $C_{l}$ is the surface concentration and $C_{0}$, the uniform concentration; $x$ is the distance along the concentration gradient and $2 l$ is the thickness of the sheet. The numerical labels for the graphs represent values of $D t / l^{2}$. The minimum time requirements for homogenization of slabs of 1 and $10 \mathrm{~mm}$ thickness at $1000^{\circ} \mathrm{C}$ are $3 \mathrm{hrs}$ and $306 \mathrm{hrs}$ and at $1400^{\circ} \mathrm{C}$ are $2 \mathrm{~min}$ and $14 \mathrm{hrs}$, respectively.

in such cases is to anneal larger specimens than actually needed for the measurements and to trim off the surface layers of the quenched samples to a depth such that the remaining core is uniform in composition.

During the anneal one must continually monitor the oxygen fugacity; this may conveniently be done by use of an electrochemical $\mathrm{ZrO}_{2}-\mathrm{Y}_{2} \mathrm{O}_{3}$ solid electrolyte cell of a design described elsewhere (Shepherd and Sandberg 1984).

\section{Additional checks of sample quality}

Before specimens are finally used in measurements it is very desirable to carry out additional checks concerning sample quality. Among these are the following:

(i) A neutron activation analysis to monitor the impurity content of the samples. Any aliovalent cations or anions present alter the $\mathrm{Fe}^{2+} / \mathrm{Fe}^{3+}$ ratio in the sample in competition with variations in oxygen stoichiometry. This effect is particularly deleterious in stoichiometric specimens $(\delta \rightarrow 0)$, as is demonstrated in figure 6 discussed below.

(ii) Powder and Laue backscattering $\mathrm{x}$-ray diffraction studies provide a check on the absence of gross inhomogeneities and show whether the specimens are indeed monocrystalline. One should note that the techniques are not suited for checking the presence of extraneous phases at levels below roughly $5 \%$ of sample mass. 


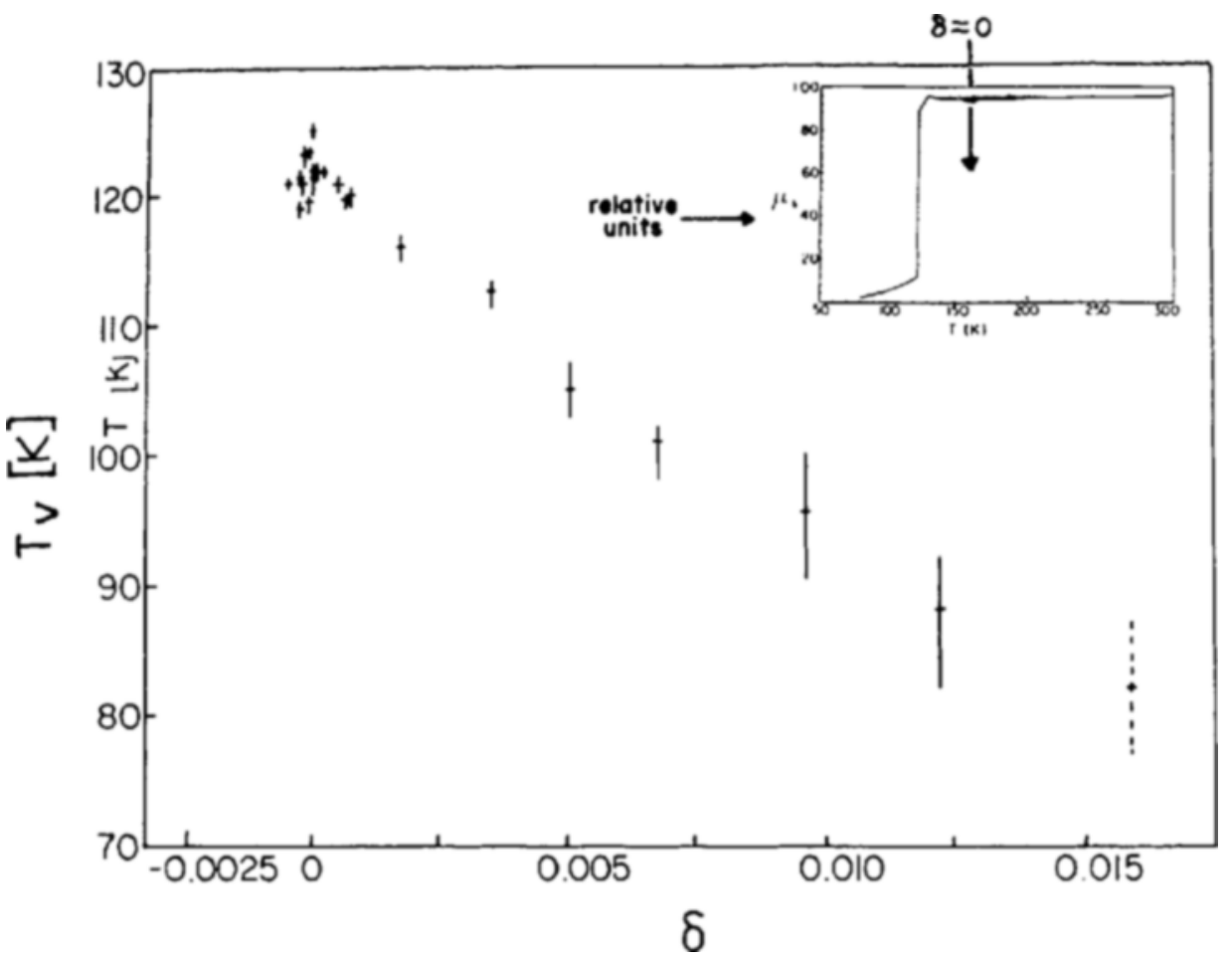

Figure 6. Variation of Verwey transition temperature with nonstoichiometry parameter. Measurements were carried out by thermomagnetic analysis, based on the measurements of initial permeability (see inset). Note the scatter of points near $\delta=0$, and the difference in data for small $(\delta \leqslant 0.004)$ and large $(\delta \geqslant 0.004)$ deviations from ideal stoichiometry $(\delta=0)$.

(iii) Polarized light microscopy is very useful in routinely detecting microscopic aggregations of phases such as wüstite or hematite in the magnetite matrix, particularly at internal grain boundaries. The degree of perfection of crystal growth as manifested by the presence or absence of voids and other large-scale imperfections can also be monitored.

\section{Experimental results: the Verwey transition temperature}

We now summarize briefly under several headings some of the work carried out at Purdue University on high quality samples produced and monitored as shown above. The first set of measurements dealt with the determination of the Verwey transition temperature. A rapid and convenient means of accomplishing this task is to perform initial magnetic permeability studies (Aragón et al 1985). The technique relies on the drastic change in the cubic anisotropy energy at the Verwey transition (Chikazumi 1975) which is then manifested in a dramatic rise of the initial permeability as the temperature $T$ is elevated past the transition temperature $T_{v}$; a typical experimental curve is shown as the inset in figure 6. Results read off from such measurements are entered in the main portion of the figure; the vertical lines show the temperature range 
near $T_{v}$ over which there is a rapid change in initial permeability in response to the comparably large change in anisotropy energy. The dot near the centre of the vertical lines represents the inflection point in the rapidly ascending permeability and closely corresponds to the Verwey temperature. It will be noted that the vertical lines in figure 6 become larger beyond a certain $\delta$ value; the reason for this observation will be discussed shortly. The principal feature of these measurements is that the transition temperature $T_{v}$ drops nearly linearly with rising $\delta$ from a high of $T_{v} \simeq 125 \mathrm{~K}$ at $\delta \simeq 0$ to a low of $T_{v}$ $\simeq 81 \mathrm{~K}$ near $\delta=0.012$. The scatter of points near $\delta=0$ arises from the presence of different amounts of impurities in the various specimens; only for $\delta>0.0005$ do the effects associated with stoichiometric variations $\delta$ outweigh those of the impurities. This provides a graphic illustration of the need to work with very pure specimens if one wishes to measure properties intrinsic to nearly stoichiometric $\mathrm{Fe}_{3} \mathrm{O}_{4}$.

One can now also understand why such a diversity of $T_{v}$ values have been quoted in the literature. In many instances workers carried out measurements on unannealed specimens and hence, on samples of unknown composition. Also, for nonuniform specimens different parts of the material may undergo the transition at somewhat different temperatures, so that the transition may seem to be broadened out.

\section{Heat capacity studies}

The Verwey transition, as any phase change, must first be understood in terms of the changes in thermodynamic properties that occur in the neighbourhood of the transformation. Accordingly, a series of heat capacity measurements were undertaken on samples of different oxygen stoichiometry. The initial objective was to settle longstanding differences in reports concerning the nature of the heat capacity anomalies that accompany the Verwey transition. In most of the measurements published earlier the anomaly extended over some twenty kelvins; for a review of this work see Shepherd et al (1985). Also, multiple peaks were frequently observed which, in turn, prompted theories that interpreted the Verwey transition as occurring in successive stages. Finally, the persistence of short-range order to temperatures far above the Verwey transition has frequently been reported.

The measurements described below were carried out on a relaxation type calorimeter (Griffing and Shivashankar 1980) in which a pulse of heat is applied and the subsequent temperature relaxation is monitored. Under appropriate operating conditions the temperature changes exponentially with time. From the rate constant of the temperature reequilibration it is possible to determine the heat capacity $C_{p}$ (Bachmann et al 1972). The advantage of this procedure is that one can carry out measurements with very small samples $(20-60 \mathrm{mg}$ in mass), so that time lag problems with large specimens are avoided. The equipment may also be operated in a transition mode procedure, whereby cooling or heating curves are taken; this permits the determination of heats of transition at $T=T_{v}$.

The results depicted in figure 7 are typical of those obtained for specimens in the composition range $-0.0002<\delta<0.004$. One encounters a single sharp spike in the heat capacity whose width, excepting the small shoulders on which the spike is superposed, does not exceed $0.4 \mathrm{~K}$. A typical heating curve for specimens in this range of $\delta$ is shown in figure 8 . It is seen that for a considerable time span the temperature changes only slightly. The total variation in $T$ over this time interval is in the 


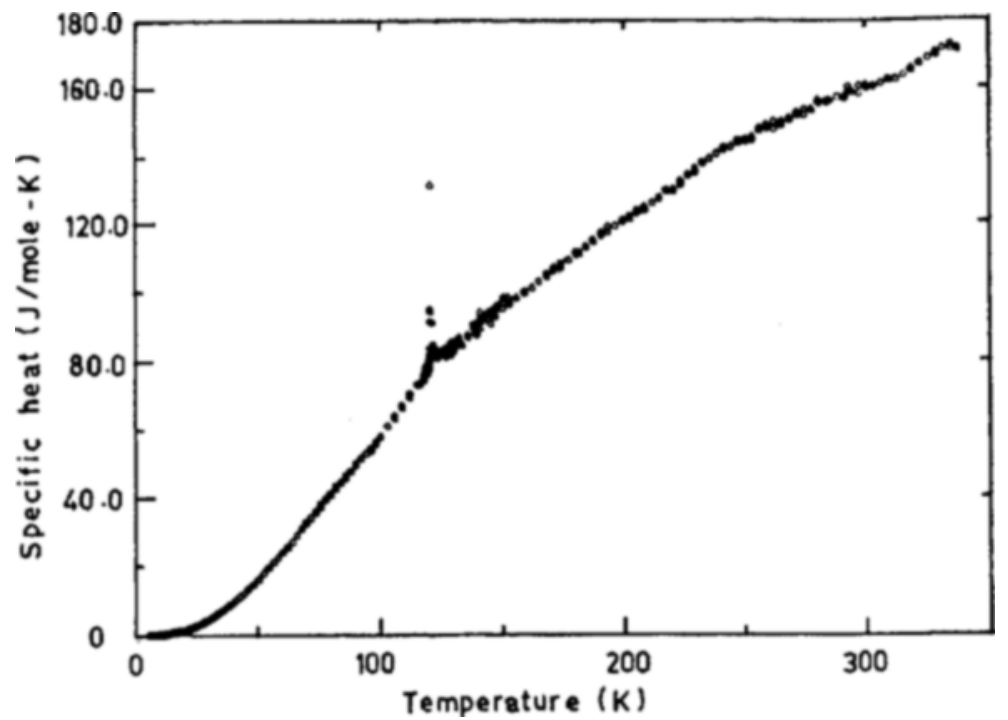

Figure 7. Heat capacity curve for a nearly stoichiometric magnetite single crystal. Note the sharp transition at the Verwey temperature.

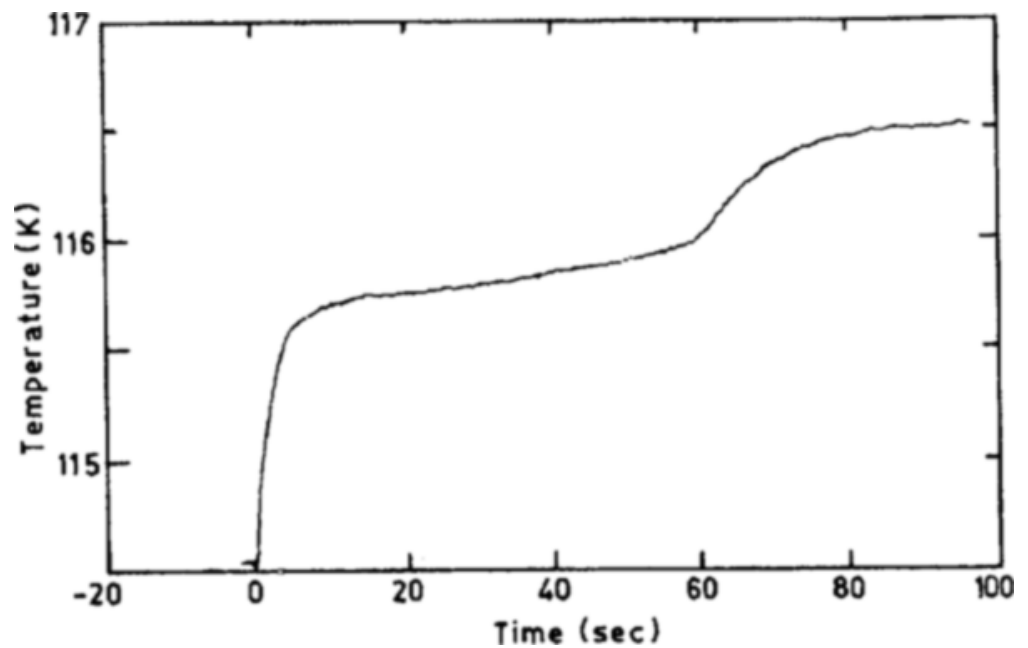

Figure 8. Heating curve for a nearly stoichiometric sample undergoing the Verwey transition. Note the temperature scale of the ordinate.

neighbourhood of $\Delta T=0.2$ to $0-4 \mathrm{~K}$, and this span diminishes as the total temperature range of the heating or cooling cycle is decreased. It appears that $\Delta T$ is actually determined by instrumental resolution problems, so that the quoted $\Delta T$ values represent upper limits.

In conformity with the results of figure 4, the transition temperature, as monitored by the position of the $C_{\mathrm{p}}$ peak, steadily diminishes with increasing departures from the 
ideal $4 / 3 \mathrm{O} / \mathrm{Fe}$ stoichiometry. The transition entropy as computed from the area under the heating or cooling curves is $\Delta S_{0}=R \ln 2$ mole $\mathrm{Fe}_{3} \mathrm{O}_{4}$ for specimens with $\delta \simeq 0$. This is half of the anticipated value if the Verwey transition involved single ion species in a sudden transition from complete charge ordering to complete disordering. This matter is further discussed below.

One of the newly discovered features in the above investigation is that in the compositional range between $\delta=0.0035$ and $\delta=0.0049$ the transition suddenly switches to a second (or higher) order regime. This is documented first by the absence of any thermal arrests in the heating curves shown in figure 10; within the noise level these

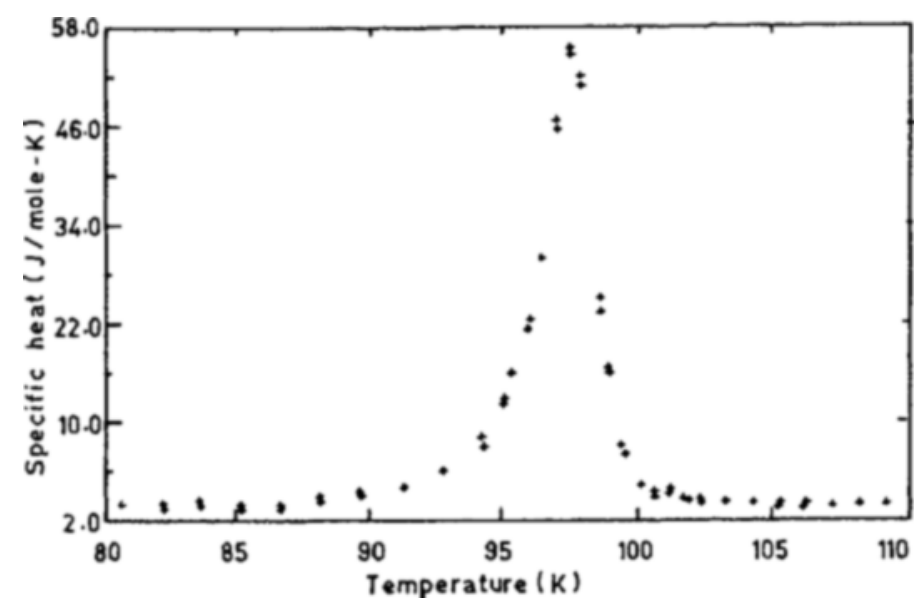

Figure 9. Excess heat capacity curve for a $\mathrm{Fc}_{3(1-\delta)} \mathrm{O}_{4}$ specimen with $\delta=0.0068$. Background curve similar to that shown in figure 7.

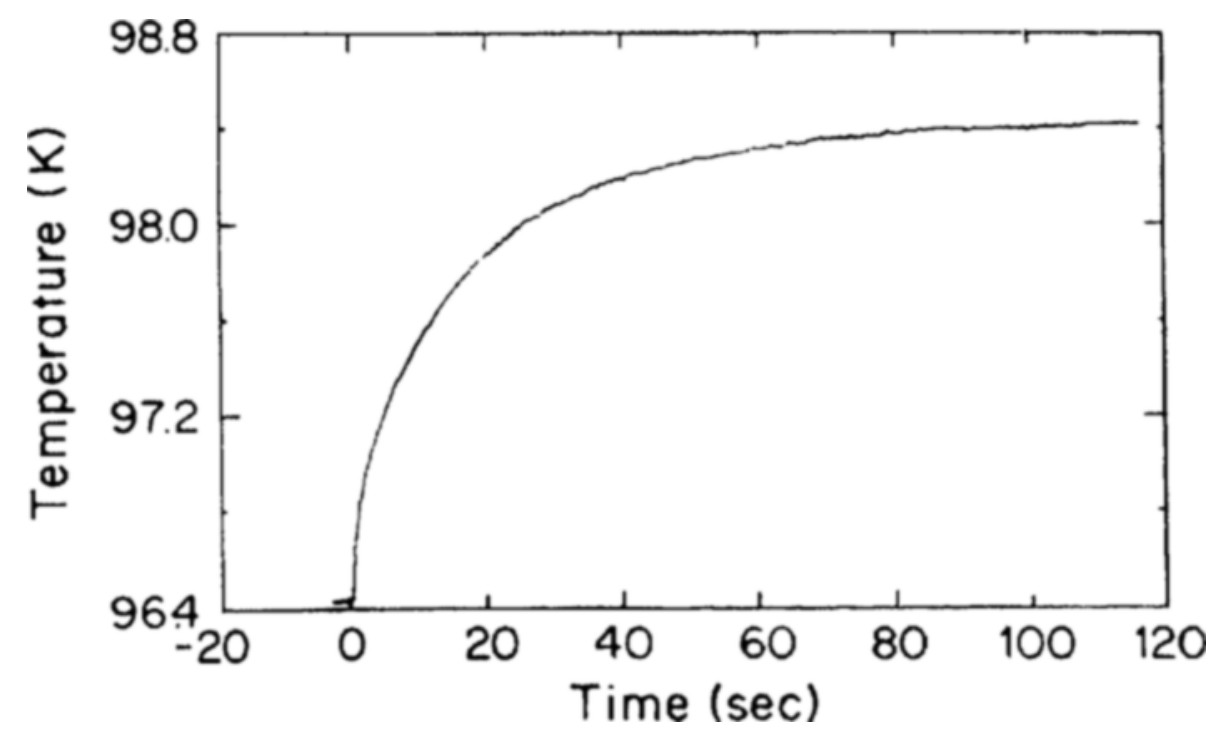

Figure 10. Thermal heating curve for sample whose heat capacity anomaly near the Verwey transition is shown in figure 9 . Within experimental error the curve is strictly exponential. 
vary strictly exponentially with time. Second, these samples exhibit a very broad, $\Lambda$-type heat capacity anomaly, as shown in figure 9, where the excess heat capacity is plotted after subtraction of the background. This broad anomaly is not simply a superposition of several first-order peaks such as might be brought about by inhomogeneities in sample composition. For, the background traces outside the temperature range of the anomalies for samples undergoing the first order transition (hereafter termed group $A$ samples) lies below the corresponding traces for the samples undergoing the second order transition (termed group B samples). Also, the heating or cooling curves for group B samples at $T=T_{v}$ are strictly exponential. The new features are demonstrated in figure 11 ; such a state of affairs persists in the temperature range $T<T_{v}$ down to the lowest measured temperatures. For $T>T_{v}$ the two types of heat capacity curves merge (or nearly merge), irrespective of whether the sample executes a first- or second-order transition to reach the cubic spinel phase. The above is in accord with the thermodynamic approach to second- (or higher-) order transitions; in principle, such an anomaly sets in at zero temperature and the deviations from the background trace grow continually until the anomaly terminates abruptly at the transition temperature. By contrast, numerical calculations of the entropy $S$ (J W Koenitzer, J P Shepherd and $P$ Wang, unpublished research) have shown that the change of $S$ with temperature $T$ for group B samples differs considerably from $S(T)$ curves for group A samples, as is shown in figure 12. The fact that for $T>T_{v}$ the heat capacity curves of the various $\mathrm{Fe}_{3(1-d)} \mathrm{O}_{4}$ specimens essentially match while the entropy curves fail to do so, suggest that electronic degrees of freedom and charge ordering processes are the important contributing factors in the Verwey transition.

One should note that only by the use of properly prepared samples was it possible to detect the subdivision of the Verwey transition into first- and second-order types and to

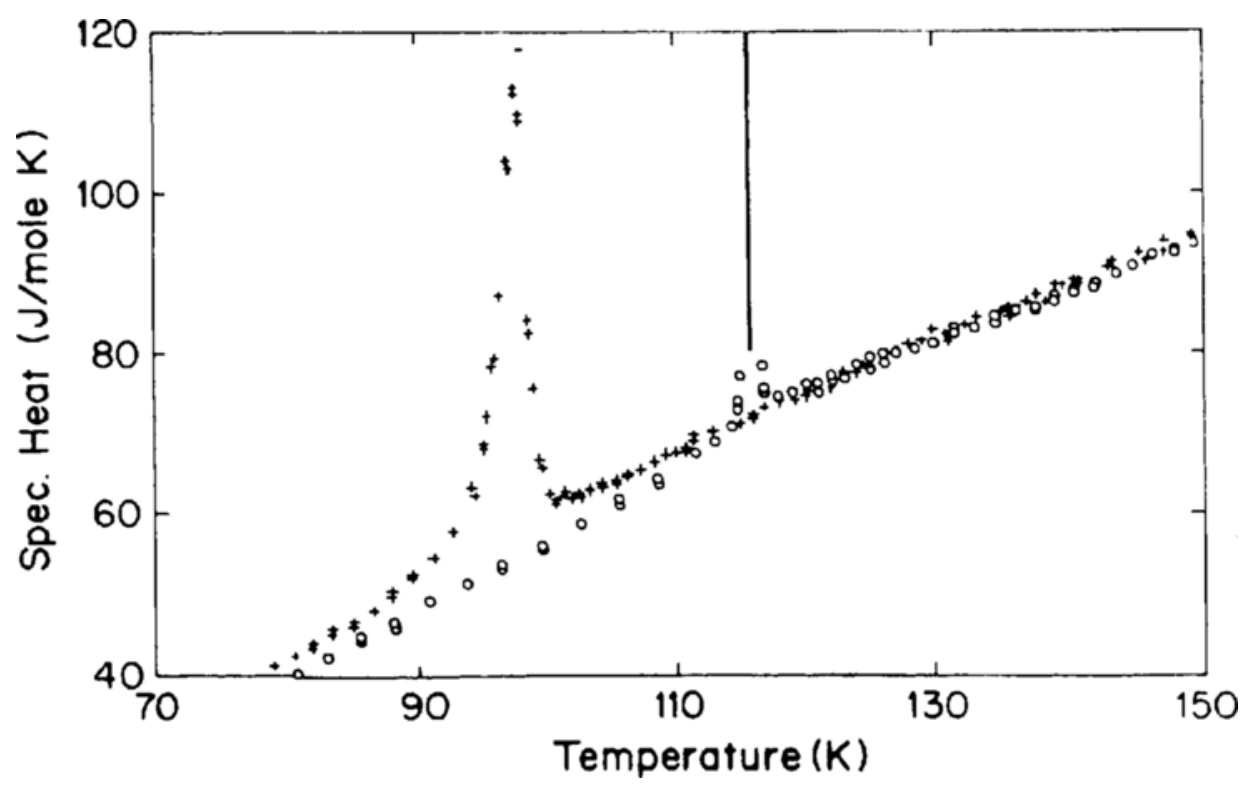

Figure 11. Comparison of heat capacity curves for two $\mathrm{Fe}_{3(1-\delta)} \mathrm{O}_{4}$ specimens with $\delta$ $=0-0017$ (right hand peak) and $\delta=0,0008$ (left hand peak). Note the matchup of the heat capacity base lines beyond $115 \mathrm{~K}$. 


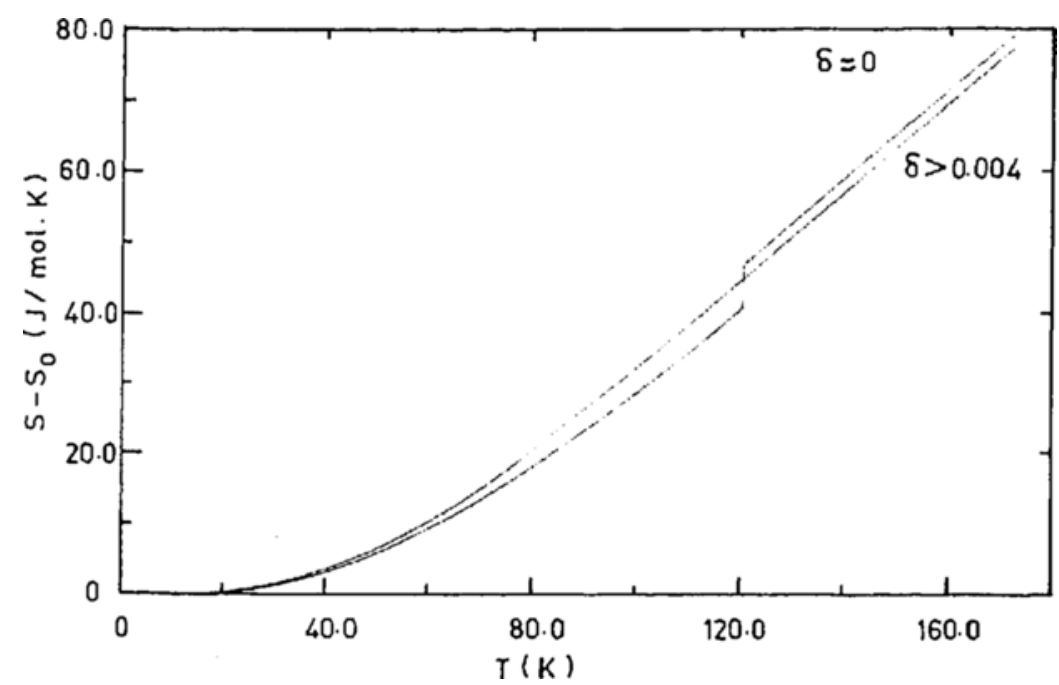

Figure 12. Entropy curves for group A $(\delta \approx 0)$ and group B $(\delta=0.0049)$ specimen as determined from heat capacity data.

achieve the very sharp set of heat capacity anomalies for the former group. Apparently, in virtually all prior measurements samples of dubious provenance were employed, so that with specimens of nonuniform composition the thermodynamic properties of magnetite could not be correctly discerned. One of the few exceptions was the research by Gmelin et al (1983) who were apparently the first to show that narrow transition ranges could be achieved in calorimetric studies and that changes in $T_{v}$ were encountered by annealing either single crystals or polycrystalline aggregates under different oxygen fugacities.

The mechanism by which $\mathrm{Fe}_{3} \mathrm{O}_{4}$ undergoes either the first or the second order transition is still undetermined. However, two quite general inferences may be drawn. The first is that the entropy change at the first-order Verwey transition is not consistent with a randomized mixing of $\mathrm{Fe}^{2+}$ and $\mathrm{Fe}^{3+}$ ions out of some elementary, ordered configuration. As stated earlier, the latter process would lead to a value $\Delta S_{v}=2 R \ln 2 /$ mole $\mathrm{Fe}_{3} \mathrm{O}_{4}$ when $\delta=0$. Rather, the observed values, $\Delta S_{v}=R \ln 2 /$ mole $\mathrm{Fe}_{3} \mathrm{O}_{4}$, may be rationalized in terms of the formation below $T_{v}$ of $\mathrm{Fe}^{2+}-\mathrm{Fe}^{2+}$ and $\mathrm{Fe}^{3+}-\mathrm{Fe}^{3+}$ dimer units in chains along the $\langle 110\rangle$ directions of the cation planes. This structure is one of several types proposed by Kita et al (1983) as being consistent with nuclear magnetic resonance and Mössbauer data. Such consistency naturally does not constitute a proof for the correctness of such an arrangement, but it does appear to rule out charge ordering at the level of single ions.

The second concerns the value of the critical $\delta_{\mathrm{cr}}$ at which the system switches from the first to the second order transition. The low-temperature monoclinic unit cell of dimension $\sqrt{2} a \times \sqrt{2} a \times 2 a$ (where $a$ is the lattice parameter of the spinel) contains 32 formula units of $\mathrm{Fe}_{3} \mathrm{O}_{4}$, i.e. $n_{\Sigma}=96$ cations. In the course of increasing the $\mathrm{O} / \mathrm{Fe}$ ratio beyond the ideal $4 / 3$ value there occurs a compensating change from the ferrous to the ferric state for some of the cations, as well as either the creation of cation lattice vacancies and/or the removal of cation interstitials according to (1)-(3). As discussed 
earlier, the density of vacancies $V$ and interstitials $I$ are related by $n_{y} n_{1} / n_{\Sigma}=K_{1}$ where $K_{1}$ is the equilibrium constant. Let us concentrate on the first of the above equations, (1): When the oxidation step has reached the stage such that one cation per unit cell has been converted from the $2+$ to the $3+$ configuration, $(3 / 8)$ of a vacancy has also been generated. The fractional change in oxygen/cation stoichiometry at that point is therefore $\delta_{c r}=(3 / 8) / 96=0-0039$, which falls comfortably in the range $0-0035$ $<\delta<0.0049$ where the changeover in transition is observed to occur.

Lastly, it is important to note that there is no indication in these thermodynamic measurements either of an extra anomalous $C_{p}$ peak at $10 \mathrm{~K}$ or of the persistence of short-range order (SRO) above $T_{v}$, which type of ordering has frequently been postulated. Careful examination of all heat capacity data in our measurements confirmed the findings of Yumoto et al (1984) who specifically searched for but failed to detect any heat anomalies in the cryogenic temperature range. Also, we do not encounter any offset in the $C_{p}$ baselines above $T_{v}$ as compared to below $T_{v}$. Thus, our thermodynamic measurements by themselves do not substantiate the existence of SRO effects.

\section{Resistivity measurements}

The question naturally arises whether the two types of thermodynamic transitions have their counterpart in electrical properties. To settle this matter it is especially important to carry out resistivity studies on appropriately grown single crystal specimens. The dangers inherent in doing such measurements on polycrystalline specimens are too well known to require elaboration here. Indeed, while the results cited below do qualitatively resemble resistivity data obtained on sintered ceramics there are significant differences on a quantitative level, and these are important when one inquires into the mechanism of charge transport in magnetite.

The change of resistivity $\rho$ with temperature $T$ is shown in figure 13 for a variety of $\mathrm{Fe}_{3(1-\delta)} \mathrm{O}_{4}$ single crystals prepared as discussed earlier (Aragón et al 1985b). This set appears to be the first truly systematic investigation of its type. Careful inspection reveals several interesting features: (i) One can clearly distinguish between group $A$ and group B samples: the former undergo a discontinuous change in $\rho$ at $T=T_{v}$; for the latter there is an anomalous rise in $\rho$ at $T=T_{v}$ but no discontinuity. Hysteresis effects are clearly in evidence for samples in group $B$. The origin of this phenomenon is unclear at this point, because one does not ordinarily associate hysteretic effects with second order transitions. (ii) In conformity with observations cited earlier, the Verwey transition temperature diminishes monotonically with rising $\delta$. (iii) The size of the resistivity discontinuity or anomaly diminishes with increasing $\delta$. (iv) The resistivity curves above $T_{v}$ essentially coincide for all samples, whereas there is a considerable, systematic spread in the curves for $T<T_{v}$, brought about by the effect mentioned in (iii); the largest resistivities are encountered for samples with $\delta \leqslant 0$. (v) Finally, it should be mentioned that these measurements have elements in common with earlier, less complete investigations on single crystals by Kuipers and Brabers $(1976,1979)$ and by several earlier investigators (Miles et al 1957; Siemon 1970; Chikazumi 1975; Terukov et al 1979). All of these latter measurements were carried on specimens sufficiently close to stoichiometry to yield discontinuities in $\rho$ at the transition point, but 


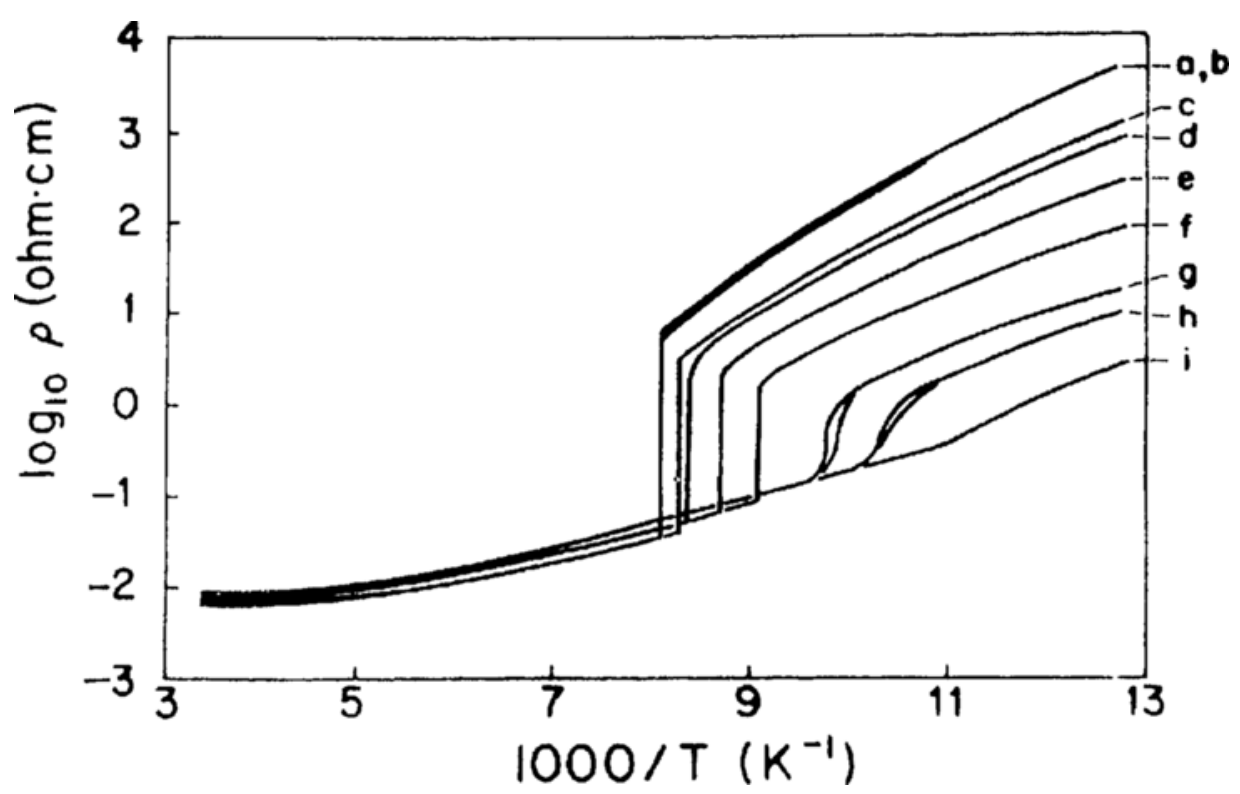

Figure 13. Resistivity of $\mathrm{Fe}_{3(1-\delta)} \mathrm{O}_{4}$ for samples with various $\delta$. Data shown as plots of $\log \rho$ vs. $1 / T$. Note the distinction between group $A$ and group B specimens. Compositions: $\delta=-0.00053$ (a), -0.00017 (b), 0.00021 (c), 0.00018 (e), 0.00069 (d), 0.0017 (e), 0.0050 (g), 0.0068 (h), 0.0097 (i).

no systematic studies linking resistivity to compositional changes had previously been reported.

There have been many attempts to provide an adequate theoretical interpretation of the electron transport properties in magnetite. These may be roughly divided into several categories: (i) Cullen and Callen $(1971,1973)$ are representatives of a school which invokes band formation and then relies on the charge ordering mechanism at the transformation temperature to open up a gap in the density-of-states originally associated with the $d$-subbands. However, in this type of approach one must rely on strong electron-electron interactions and/or on quite small orbital overlaps between the constituent atoms to achieve a band-width small enough for the rather small charge carrier mobilities cited below. This avenue was carefully explored by Ihle and Lorenz (Ihle 1984; Ihle and Lorenz 1974, 1980; Lorenz and Ihle 1975a, b, 1979, 1982), who invoke SRO effects as a complication. The destruction of SRO with rising temperature simulates thermally activated conduction processes. As we stressed earlier, however, such SRO effects and their destruction have not been detected in our heat capacity measurements. In such circumstances it might be more appropriate to start out with a small-polaron (hopping) model of charge carriers. (ii) Srivastava (1983) as well as Hurd and collaborators (McKinnon et al 1981; Shiozaki et al 1981) have introduced an incoherent phonon-driven tunnelling mechanism to explain the observed electrical transport phenomena; the difficulty with this approach is that very unrealistic values of the various physical parameters must be introduced to obtain agreement with experiments. (iii) A very commonly championed view is that charge transport occurs via 
the small polaron mechanism. This is in large measure supported by the Seebeck measurements cited below, but a relatively persuasive, independent argument is provided through the simple expression $\sigma=n e u$, relating the conductivity $\sigma \equiv 1 / \rho$ to the density of charge carriers, $n$, their electric charge $e$, and their mobility $u$. Even for the extreme case $T=300 \mathrm{~K}$, where $\sigma \simeq 10^{2} \mathrm{ohm}^{-1} \mathrm{~cm}^{-1}$, one requires a mobility $u$ $\simeq 0.5 \mathrm{~cm}^{2} / v$-sec for charge carrier densities $n \simeq 10^{22} \mathrm{~cm}^{-3}$. Admittedly, this figure is close to the upper limit (Rathenau and Goodenough 1968) of mobilities associated with thermally activated charge transport, but for temperatures $T<250 \mathrm{~K}$ the conductivity has already become sufficiently small such that the requisite probabilities fall well below this upper limit. $A$ forterior $i$ this is even more the case below the Verwey transition temperature. One argument occasionally advanced against the small polaron mechanism is that data plots of the type shown in figure 13 do not form straight lines, such as are frequently encountered in the range $T<T_{v}$ for ceramic or polycrystalline material. However, contrary to misconceptions frequently quoted in the literature, departures from the Arrhenins behaviour do not signal a breakdown of the small polaron model. Rather, if one accepts charge transport as occurring by thermally activated displacement of carriers then conventional theory (Holstein 1954; Reik and Heese 1967) predicts a mobility $u(T)$ with a temperature dependence of the form

with

$$
u \propto(\tau / T) \exp \left\{-\eta \tan \mathrm{h}\left(\hbar \omega_{0} / 4 k T\right)\right\},
$$

$$
\tau^{2} \equiv\left[\sinh \left(\hbar \omega_{0} / 2 k T\right)\right] / 2\left(\hbar \omega_{0} / k\right)^{2} \eta,
$$

in which there are two disposable parameters; the appropriate optical lattice frequency $\omega_{0}$ and the degree of coupling $\eta$ of the charge carriers to the lattice (roughly, the number of polarons clothing the electron or hole). These parametric degrees of freedom suffice to provide the needed curvature for a reasonable fit of the above equation to the data in figure 13. Only as an extreme limiting case does one obtain the usually-cited result $\ln u=-\hbar \omega_{0} / 2 k T+$ constant, which is clearly inapplicable here. (iv) Lastly, a combination of small polaron band and hopping processes has been recently suggested to treat the electrical transport phenomena (Ihle and Lorenz 1985). Again, the gradual disappearance of SRo with increasing $T>T_{v}$ was invoked, and this once more raises the questions as to the actual existence of short-range order configurations above $T_{v}$.

\section{Seebeck coefficient measurements}

We conclude with a brief summary of Seebeck coefficient $(\alpha)$ measurements. The data collected in the author's laboratory (Aragón et al 1985) are displayed in figure 14 as plots of $\alpha$ vs $T$. These results are similar in form to a more limited set published by Kuipers and Brabers $(1976,1979)$ on single crystals of magnetite or titanomagnetite containing small concentrations of $\mathrm{Ti}^{4+}$ close to ideal stoichiometry. Again, the data obtained here differ significantly from measurements reported for polycrystalline samples or ceramic types. Several features are noteworthy: (i) One may readily distinguish between the regions $T>T_{v}$ where the various Seebeck coefficients are nearly independent of temperature, and the region $T<T_{v}$ where $\alpha$ changes considerably with $T$. The near-constancy of $\alpha$ in the high temperature regime is difficult to reconcile with a band model involving itinerant charge carriers except for the special 


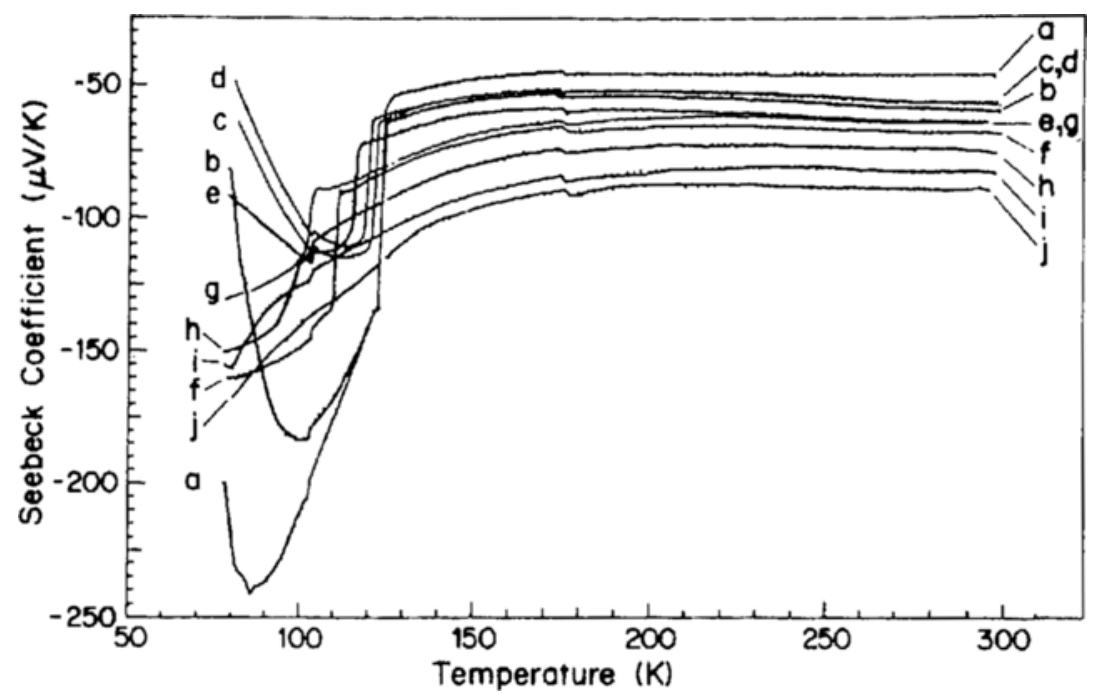

Figure 14. Secbeck coefficient data ( $\alpha$ ) for $\mathrm{Fe}_{3(1-\delta)} \mathrm{O}_{4}$ single crystal specimens with various $\delta$. Data shown as plots of $\propto$ vs. T. Compositions: $\delta \simeq 0(\mathrm{a}, b), 000017$ (e), 0.00069 (a), $0.0018(\mathrm{e})$, $0.0036(f), 0-0052(\mathrm{~g}), 0.0070(\mathrm{~h}), 0-0099(\mathrm{i}), 0-012(\mathrm{j})$. Note the distinction between group $A$ and group B specimens.

case of an extrinsic band-type semiconductor for which the exhaustion range limit has been reached. However, the $\alpha$ values for such cases tend to be much larger than the values shown in figure 14. A much more natural explanation involves the use of the Heikes equation in the form (Van Zandt and Honig 1981)

$$
\alpha \equiv-(k / e) \ln \left\{2\left[\mathrm{Fe}_{B}^{3+}\right] /\left[\mathrm{Fe}_{B}^{2+}\right]\right\},
$$

where $k$ is Boltzmann's constant and $\left[\mathrm{Fe}_{B}^{3+}\right]$ denotes the concentration ferric ions on $B$ sites. For strictly stoichiometric material, $\alpha=-(k / e) \ln 2=-60 \mu \mathrm{V} / \mathrm{deg}$, in reasonable agreement with the observed values. The constant $\alpha$ values shown in figure 14 change with $\delta$ roughly in conformity with (13), whereas Kuipers and Brabers $(1976,1979)$ encountered no such dependence. (ii) For $T>T_{v}$ the samples in group A undergo a discontinuity in $\alpha$ which moves to lower temperatures with increasing departures from ideal stoichiometry; the samples in group B exhibit an increasing dependence of $\alpha$ on $T$ commencing at roughly $120 \mathrm{~K}$. (iii) For $T<T_{\mathrm{v}}$ the samples in group A pass through a minimum in $\alpha$ and then become less negative as the temperature is dropped. The samples in group B show a further decrease in $\alpha$ as $T$ is diminished. These samples ultimately also must pass through a minimum since, by the third law of thermodynamics, $\alpha$ must come into the origin of coordinates with zero slope as $T \rightarrow 0$. Kuipers and Brabers (1976) reported a change in sign in $\alpha$ as the temperature is lowered, in contrast to the present findings (although such a change in sign might still be encountered at temperatures below the current set of measurements). They interpret their findings (similar to our results for group A specimens) on the basis of a two-carrier model involving small polarons of positive (hole-like) and negative (electron-like) charge. 
Again, one should note that the results obtained with well-annealed single crystals differ qualitatively with those published in the literature on compacted polycrystalline specimens. This once more illustrates the problems inherent in the presence of grain boundaries and internal contacts in the latter group of samples.

\section{Concluding comments}

As has been repeatedly stressed, it is absolutely essential to develop adequate techniques for preparation and characterization of $\mathrm{Fe}_{3} \mathrm{O}_{4}$ samples in order to ensure that the various experimental measurements yield reliable results. Failure to appreciate this point in the past has led to confusion and to the publication of contradictory data in the literature. For thermodynamic and transport data the difference in experimental findings between adequately and inadequately prepared samples may be readily documented. It may equally well turn out that structural problems revealed by $x$-ray diffraction, electron diffraction, neutron diffraction, Mössbauer, or nuclear magnetic resonance measurements can be settled only after extensive reinvestigations with properly prepared specimens.

\section{Acknowledgments}

This research was supported by a grant from NSF-DMR 8402864 AO1. The author is greatly indebted to Dr R Aragón for his critical readings of the manuscript. He wishes to take this opportunity to thank his colleagues R Aragón, D J Buttrey, H R Harrison (now deceased), J Koenitzer, M Pai, R Rasmussen, C J Sandberg, and J P Shepherd for their excellent, dedicated, and enthusiastic research efforts that resulted in the work described above.

\section{References}

Aragón R, Buttrey D J, Shepherd J P and Honig J M 1985a Phys. Rev. B31 430

Aragón R, Shepherd J P, Koenitzer J W, Rasmussen R J and Honig J M 1986 J. Magn. \& Magn. Mater. 54-57 1335

Bachmann R, Di Salvo F J, Geballe T H, Greene R L, Howard R E, King C N, Kirsch H C, Lee K N, Schwall R E, Thomas H V and Zubeck R B 1972 Rev. Sci. Instrum. 43205

Berzelius J J 1826 Lehrbuch der Chemie (Dresden) Vol. 2, p. 360

Chikazumi S 1975 AIP Conf. Proc. 29382

Chikazumi 1976 in Magnetism and magnetic materials: Proc. 21 st Annual Conf. on magnetism and magnetic materials (eds) J J Becker, G H Lander and J J Rhyne (New York: AIP) p. 382

Cullen J R and Callen E 1971 Phys. Rev. Lett. 26236

Cullen J R and Callen E 1973 Phys. Reu. B7 397

Dalton J 1816 Ann. Chim. Phys. 1472

Dieckmann R 1982 Ber. Bunsenges. Phys. Chem. 81112

Dieckmann R and Schmalzried H 1977 Ber. Bunsenges. Phys. Chem. 81344

Flood H and Hill D G 1957 Z. Elektrochem. 6118

Gay-Lussac L J 1811 Ann. Chim. (Paris) 80163

Gay-Lussac L J 1812 Gilberts Annal. 42166

Gmelin E, Lenge N and Kronmüller H 1983 Phys. Status Solidi A79 465

Griffing B F and Shivashankar S A 1980 Rev. Sci. Instrum. 511030 
Harrison H R and Aragón R 1978 Mater. Res. Bull. 131097

Harrison H R, Aragón R, Keem J E and Honig J M 1983 in Inorganic Syntheses (ed.) S L Holt (New York: Wiley \& Sons)

Holstein T 1954 Ann. Phys. (N.Y.) 8325

Honig J M $1982 \mathrm{~J}$. Solid State Chem. 451

Ihle D 1984 Phys. Status Solidi B121 217

Ihle D and Lorenz B 1974 Phys. Status. Solidi B63 599

Ihle D and Lorenz B 1980 Philos. Mag. B42 337

Ihle D and Lorenz B 1985 J. Phys. C18 L647

Kita E, Tokuyama Y, Tasaki A and Siratori K 1983 J. Magn. \& Magn. Mater. 31-34 787

Kuipers A J M and Brabers V A M 1976 Phys. Rev. B14 1401

Kuipers A J M and Brabers V A M 1979 Phys. Rev. B20 594

Lorenz B and Ihle D 1975a Phys. Status Solidi B68 K189

Lorenz B and Ihle D 1975b Phys. Status Solidi B69 451

Lorenz B and Ihle D 1979 Phys. Status Solidi B96 659

Lorenz B and Ihle D 1982 Phys. Status Solidi B113 601

McKinnon W R, Hurd C M and Shiozaki I 1981 J. Phys. C14 L877

Miles P A, Westphal W B and von Hippel A 1957 Rev. Mod. Phys. 29279

Mott N F 1979 Festkörpeprobleme 19331

Mott N F 1980 Philos. Mag. B42 327

Néel L 1948 Ann. Phys. (Paris) 3137

Okamura T 1932 Sci. Rep. Tohôku Imperial Univ. 21231

Parks G S and Kelley K K $1926 J$. Phys. Chem. 3052

Rathenau G W and Goodenough J B 1968 J. Appl. Phys. 39403

Reik H G and Heese D 1967 Phys. Status Solidi 24281

Shepherd J P, Kocnitzer J W, Aragon R, Sandberg C J and Honig J M 1985 Phys. Rev. B31 1107

Shepherd J P and Sandberg C J 1984 Rev. Sci. Instrum. 551696

Shiozaki I, Hurd C M, McAlister S P, McKinnon W R and Strobel P 1981 J. Phys. C14 4641

Siemon W J 1970 IBM J. Res. Dev. 14245

Srivastava C M 1983 Bull. Mater, Sci. 5247

Terukov E I, Reichelt W, Ihle D and Oppermann H 1979 Phys. Status Solidi B95 491

Van Zandt L L and Honig J M 1981 J. Appl. Phys. 525625

Verwey E J W 1939 Nature (London) 144327

Yumoto S, Mizoguchi M and lida S 1984 J. Phys. Soc. Jpn 5326 